\title{
Improving Perceptual Multimedia Quality with an Adaptable Communication Protocol
}

\author{
Gheorghita Ghinea ${ }^{1}$ and Johnson P. Thomas ${ }^{2}$ \\ ${ }^{1}$ Department of Information Systems and Computing, Brunel University, Uxbridge, UK \\ 2 Department of Computer Science, Oklahoma State University, Tulsa, USA
}

\begin{abstract}
Innovations and developments in networking technology have been driven by technical considerations with little analysis of the benefit to the user. In this paper we argue that network parameters that define the network Quality of Service (QoS) must be driven by user-centric parameters such as user expectations and requirements for multimedia transmitted over a network. To this end a mechanism for mapping user-oriented parameters to network QoS parameters is outlined. The paper surveys existing methods for mapping user requirements to the network. An adaptable communication system is implemented to validate the mapping. The architecture adapts to varying network conditions caused by congestion so as to maintain user expectations and requirements. The paper also surveys research in the area of adaptable communications architectures and protocols. Our results show that such a user-biased approach to networking does bring tangible benefits to the user.
\end{abstract}

Keywords: adaptable communication protocols, perceptual quality, multimedia, Quality of Service.

\section{Introduction}

The concept of Quality of Service in distributed multimedia systems is indelibly associated with the provision of an acceptable level of application performance. Ultimately, this performance is itself dependent on

1. the user's experience with the multimedia presentation which we define as Quality of perception (QoP). QoP has two main components: a user's ability to analyse, synthesise and assimilate the informational content of multimedia applications, as well as his/her subjective satisfaction with the quality of such applications.
2. the service provided by the underlying network.

Such a user-biased multimedia system would be fundamentally based on a mapping linking user-centric QoP to low-level QoS parameters. Appropriate management of QoS parameters provides the potential of ensuring an optimum user experience in a distributed multimedia setting. The networking foundation on which current distributed multimedia applications are built, either do not specify QoS parameters (also known as best effort service) or specify them in terms of traffic engineering parameters such as delay, jitter, and loss or error rates. However, these parameters do not convey application-specific needs such as the influence of clip content and informational load on the user multimedia experience. There is thus an architectural gap between the provision of network-level QoS and application-level usercentric requirements of the distributed multimedia applications. This gap causes distributed multimedia systems to inefficiently use network resources and results in poor end-to-end performance which in turn has a direct negative impact on the user experience of multimedia. In this paper, we review previous work done in both these domains. This includes previous approaches to map user-centered preferences to network parameters. We propose and implement an integrated approach to this problem. In our approach, we first map the user-centered parameters of a multimedia presentation to network QoS variables. We then review existing QoS oriented communication architectures and protocols. Our mapping is implemented on 
an adaptable communication architecture which enables protocols to adapt in order to optimize the user experience of the multimedia presentation, even as the underlying network services change due to congestion. Our results show that the mapping-based adaptable architecture does indeed provide a better experience for the user in terms of perception/enjoyment when compared to architectures that do not cater for the user.

\section{Bridging the Gap: from User Quality of Perception to Quality of Service}

\subsection{Introduction}

There have been few instances in the literature of research being done in the area of bridging the application-network gap. This probably reflects the inherent difficulty of trying to link subjective sentiments about the quality of the presentation with the facts and figures of network parameters.

In essence, there are three approaches that can be identified:

1. In the implicit approach, there is no explicit mapping between application-level user requirements and the QoS provided by the network. The user specifies, usually through a Graphical User Interface, his/her desired presentation quality, for example, the desired playback frame rate or spatial resolution.

An experimental test-bed for delivering multimedia over the World Wide Web with a user-specified QoS has been developed in [1]. The user specifies his desired video quality, which includes various frame rates, large or small video size, as well as the encoding mechanism (JPEG or H.261). The user can also indicate a priority for these QoS parameters, as well as the hardware capabilities (e.g. CPU, Video Hardware) of the host machine. A QoS degradation path is computed. The QoS degradation path is an ordered list obtained according to the user-specified QoS preferences according to which the delivered QoS incrementally varies as a result of fluctuating network conditions.

The Total Management Of Transmissions for the End-user (TOMTEN) framework for user management of end-system and network resources [2] framework is reactive as it is only active when the application is started or the user indicates dissatisfaction with the multimedia quality of a session. This is in contrast to most QoS models which involve a predictive (bandwidth estimation) approach [3]. After starting a TOMTEN session, the user is asked to specify the general importance of the application (high, medium or low), upon which the system checks available system resources and suggests a short list of the most appropriate configurations to try to satisfy user requirements.

In [4], the approach to bridging the application-network gap is based on a 'softness' profile. Essentially, the less variation in user satisfaction with an application in response to a relatively much greater variation in bandwidth, the 'softer' the application's QoS profile is. Thus, the softer an application's profile is, the easier it is for it to degrade gracefully (from an end-user satisfaction perspective) under increasing network load. For Video on Demand (VoD) applications, the user indicates the name of the video server, his/her desired movie, as well as the associated priority of a media object (Importance of Presence) and the coding quality (quantization, pixel resolution, and frame rate) of a media object (Level of Detail) through sliders of a GUI. During the viewing process, in response to network load changes, QoS reconfiguration may take place, whereby the user may desire to readjust QoS parameters with the sliders provided. If this new QoS configuration is not acceptable, then the server provides feedback in the form of visual adjustment of the user's selected values, thus indicating the feasible operational range.

2. In the second approach, an explicit mapping linking application-level user requirements to network QoS is actually given. Such a mapping can either be defined on a per layer basis (such as a network to transport to session to application-layer mapping), or directly between application and network-level parameters. The problem here is two-fold: the number of layers in question (this depends on the communication architecture) and of comprehensively providing maps across all layers. To the best of our knowledge, the latter has not been 
achieved. What is usually done is to provide an application-transport layer mapping, and to let network layer issues be handled by mechanisms such as the Resource Reservation Protocol (RSVP) or Q.2963.3 signalling (in the case of ATM networks). Such an application-transport layer mapping is proposed in [5]. Unfortunately, their paper is mainly conceptual, although results using the mapping are subsequently reported in [1]. Furthermore, it does not treat the important problem of per-application QoS requirements.

3. The last approach is, in essence, a more restrictive version of the first. What happens here is that the user is played short-duration probes of differing qualities of the multimedia material in question and $(\mathrm{s})$ he then specifies which of the given sample qualities is acceptable. Apart from the obvious goal of polling user-preferred multimedia quality, the probe-based approach is advantageous from the point of view that it tests current network conditions.

In the Quality Query by Example [6], the user is typically presented with sample qualities of the requested viewing material prior to viewing it properly. Such samples can come in the form of images (or short video sequences) of different size, resolution, colour depth and frame rates (in the case of video) coupled with speech of, say, either telephone or CD quality. During this probing period, via the means of a slider, the user can change the quality of the viewed material till an acceptable one is found. The advantages of this method are twofold: firstly, the user is not asked to specify a whole lot of technical terms (such as those specified in implicit mappings) with which (s)he might be unfamiliar, thereby providing a more comfortable interface for specifying the desired quality; secondly, the user can actually see in front of her the whole range of quality with which the presentation can be delivered, rather than a test-and-see approach that is characteristic of other types of mappings.

An on-line, probe-based protocol has been developed in an attempt to overcome the problem of pessimistic, worst-case estimates used in QoS negotiation [7]. Their approach determines a realistic value for a QoS parameter of interest (e.g. video frame rate) and identifies a degradation point over a specific time interval I, when performance starts to degrade due to buffer problems and mismatched rates between the server and client.

\subsection{Mapping}

The delivered QoS is influenced by parameters such as jitter, delay, bit error rate, segment loss and segment order. Making allowance for any operating system overheads, it is precisely the value of these parameters which determines the actual display frame rate of a multimedia clip or the visual effect of its colour palette and ultimately the user experience.

Although the various protocol stacks (such as $\mathrm{TCP} / \mathrm{IP}$, UDP/IP) manage these parameters in one form or another, this is done with purely a technical scope (e.g. the maintenance of the parameter within certain bounds), totally ignoring the impact that it might have on the user. For instance, one would expect that users' subjective quality assessment of multimedia clips should be quite different for a clip viewed at 25 frames per second (fps) with a 24-bit colour depth from the one viewed at $15 \mathrm{fps}$ with an 8bit colour depth. In fact, our previous work has shown is that, on average, the difference in quality is barely noticeable, as is the content assimilation [8]. This leads to the conclusion that management of these low-level network QoS parameters should include, at the very least, considerations upon their impact on user-level QoP. In other words, given a set of QoS parameters how must they be managed in order to maximise user-level QoP?

\section{Approach Outline}

There is an inherent difficulty in obtaining a mapping between the user's high-level, subjective, QoP and the low-level network parameters. Although a direct mapping cannot be currently obtained, we can specify a relation of proportionality between QoP and QoS. As our primary interest is in increasing QoP through appropriate management of QoS resources, such a relation will be sufficient for our purposes. Details of our QoP-QoS mapping can be found in [9].

Our previous research has shown that QoP varies strongly with the informational complexity of multimedia application being visualised, where, 
by informational complexity of a multimedia clip, we understand the relative importance of the video, audio and textual components of a clip as purveyors of information [8]. We can therefore conclude that QoP is directly proportional to the quality with which the main conveyor of information is delivered in the context of the clip.

A coarse mapping can be obtained linking the QoS of the respective media, QoSVideo, QoSAudio and QoSText to low-level network QoS parameters. In our work we have concentrated on: bit error rate, delay, jitter, segment order and segment loss. This mapping can be specified through a matrix (Table 1), where the importance of the low-level QoS network parameters on the delivered QoS of the respective informational components is given by three discrete levels (low, medium, high).

\begin{tabular}{|c|l|c|c|c|}
\hline \multicolumn{2}{|c|}{$\begin{array}{l}\text { QoP To QoS } \\
\text { MAPPING }\end{array}$} & \multicolumn{3}{c|}{ QoP } \\
\cline { 2 - 5 } & VIDEO & AUDIO & TEXT \\
\hline \multirow{4}{*}{ Q } & Bit error rate & low & low & low \\
\cline { 2 - 2 } o & Delay & medium & medium & low \\
\cline { 2 - 2 } S & Jitter & medium & low & medium \\
\cline { 2 - 2 } & Segment loss & low & high & high \\
\cline { 2 - 2 } & Segment order & high & medium & medium \\
\hline
\end{tabular}

Table 1. Conversion Matrix linking QoP to QoS.

The envisaged QoP to QoS mapping will therefore be media specific. Thus, if the user attaches importance to the textual component of a multimedia application (such as those encountered in collaborative environments) then, although individual bit errors may be tolerated, loss of contiguous frames will not. The human brain's ability to filter noise and reconstruct missing information means that, even if bit errors do occur, perception and understanding will not suffer proportionally. However, the loss of a series of frames will result in a volume of the text being displayed for a much shorter length of time than intended or not at all. This is less tolerable than individual bit errors. Table 1 reflects this situation, whereby bit error rates are deemed to be of low importance to the textual component of a multimedia application, while the loss of a segment is considered to have a high importance vis a vis the same media component. If the video stream has been identified as having high priority, then, as indicated in Table 1 , bit error rate is of low importance to the video component of the presentation whereas segment order is more crucial. As far as audio is concerned, it is imperative that packets are not lost. Therefore, if one preferentially manages these network QoS parameters (as dictated by their associated weights), then chances are that QoP will be improved.

We illustrate the ideas behind the mapping with an example. Let us assume that we have a multimedia clip where the values of $\mathrm{V}, \mathrm{A}$ and $\mathrm{T}$ have been judged to be high, medium and low, respectively. In other words, the informational load being conveyed by the video component is high, that delivered by the audio stream is medium, while the one delivered by the textual component is low.

Let the three discrete levels of low, medium and high of V, A and T, as well as the elements of Table 1, be mapped onto the numeric values of 1,2 , and 3 , respectively. Then, by introducing these values into our mapping [9] we get:

$$
Q o P_{I T} \cong B E R+\frac{11}{6} D E L+\frac{10}{6} J I T+\frac{5}{3} S L+\frac{5}{2} S O
$$

What this last relation shows is that the QoS parameter of highest importance to the application is the segment order (since it has the highest coefficient, 2.5) followed by delay, jitter, segment loss and bit error.

Moreover, (1) reflects the spread of informational load in the clip. If the vector-distances between the vector on the right hand side of (1) and the three vectors corresponding to the three columns of V, A and T in Table 1 are computed, these distances show a bias towards the video, audio and textual components (in this order) of the clip, which accurately reflects their high, medium and low importance, respectively, as conveyors of information.

\section{Multimedia Communication Mechanisms}

In the previous section we argued that the underlying QoS parameters have an impact on userlevel QoP and a QoP - QoS mapping was outlined. To validate our mapping an adaptable communication system was implemented. We 
now review work on adaptable communication protocols for multimedia QoS. This will lead to the validation of our mapping.

The layered communication architecture based on the OSI reference model, as well as many of the network protocols in use today, are ill-suited for supporting distributed multimedia applications. Traditional protocols such as TCP/IP were conceived at a time when the emphasis was laid on providing functionality for data transfer over unreliable networks and not for satisfying the diverse requirements of multimedia applications. While the OSI reference model has a number of QoS parameters describing the speed and reliability of transmission, these parameters apply to lower protocol layers and are not meant to be directly observable or verifiable by the application. Legacy communication architectures provide no support for negotiation and maintenance of applications' QoS, nor for its re-negotiation should the applications' requirements change. Moreover, there is no facility for the reservation and allocation of system and network resources needed by multimedia applications.

In order to meet the broad range of multimedia QoS requirements and to take full advantage of the services provided by the underlying networks, it is necessary that new approaches be elaborated for the distributed multimedia applications of the future.

One approach is to modify legacy protocols to be made more suitable for distributed multimedia applications. However, even such optimisations do not provide the flexible functionality required by multimedia applications. Therefore, totally new protocols and communication architectures need to be elaborated. Both will have adaptivity as a sine qua non requirement.

\subsection{Adaptivity in Multimedia Communications}

An important feature of multimedia applications is that they have a wide spectrum of dynamically varying QoS requirements which must be negotiated, re-negotiated and managed in response to changing network and end-system conditions, or to new expectations from the human user. Thus in a distributed multimedia QoS context, it is precisely this (re)negotiation and dynamic management of applications' QoS that emphasises the need for adaptable protocols protocols that are capable of modifying their execution pattern to suit their changing environment [10].

In essence, there are two approaches to the development of new protocols for distributed multimedia applications. In the first approach multimedia protocols are realised with adequate communication architecture support. In this case, protocols are developed as part of a suite within the confines of new communication architectures. In the second approach protocols are elaborated on a standalone basis and deal with QoS issues at a specific layer in the understanding that they will form, together with either legacy protocols or other new protocols (addressing QoS issues at other layers), new protocol stacks capable of QoS support.

\subsection{Adaptable Communication Architectures and Protocols for Multimedia QoS}

A Quality of Service (QoS) management architecture for distributed multimedia applications in heterogeneous communication environments of wired and wireless networks is described in [11]. A proxy server called Communication Coordination Server (CCS) is proposed, which intermediates a video server and a receiver and manages the QoS coordination. The CCS performs QoS admission, adjustment, and allocation mechanisms to satisfy the user's QoS requirement. Transcoding is used to realize the allocated QoS, and it decodes the input video stream from the video server and encodes it within the CCS. A QoS mapping mechanism translates application-level QoS into resourcelevel QoS for the QoS admission. They also propose a new QoS mapping mechanism using spline functions that enable a continuous QoS translation.

A conceptual framework for adaptation has also been proposed in [12]. It is suggested that altering or adapting the data flow in various ways at some or all points in the network could benefit the sender, receiver, and network administrator. Different kinds of adaptation agencies (AA), which range from adaptive protocols to heavyweight code, alter or adapt the data flow. An AA consists of an event manager that monitors the environment, that is, traffic, error conditions, and CPU cycles on a local processor. The event 
manager can receive control messages which alter the behaviour of the AA. The Resource Management and Monitor (RM) component handles resources under direct control of the AA. If the AA has been allocated a certain percentage of a data link's bandwidth, the RM determines how to best use that bandwidth to meet the needs of all data flows under its control. Each AA may contain zero or more Application Specific Adapters (ASA) which perform some particular adaptation on a data stream. However, this is a conceptual framework and has not been implemented.

The fittingly entitled ADAPTIVE (A Dynamically Assembled Protocol Transformation, Integration and Validation Environment) project provides an integrated environment for protocol composition, integration and experimentation [13]. The configuration of a protocol is tailored at connection set-up time, based on the multimedia application's required QoS and the prevailing network conditions. The environment parameters which determine the initial configuration of the protocol are traffic volume, intermediate switching node queue lengths, host processing loads and services available at the remote hosts. These parameters are negotiated by host-based agents. ADAPTIVE will ensure that the generated protocols respond to changes in application requirements or network characteristics, either by explicit or implicit dynamic reconfiguration of the transport session. ADAPTIVE also provides the possibility of using 'plug-in' protocol components such as timers, message buffers and protocol graph operations that insert, delete and alter protocol objects.

A different perspective is taken in [14] who propose a scalable closed-loop traffic management protocol called Multiplex Adaptive Queuing (MAQ) to adaptively maintain QoS. At edges of a network users want fine-grain QoS granularity whereas inside a network only coarsegrain granularity is achievable. For example, IP/MPLS networks do not support per-connection QoS, whereas users would expect per-connection QoS. MAQ assumes a small number of priority classes and queues inside the core network and supports a large number of QoS classes at the edge. The large numbers of QoS classes are then mapped onto core network dynamically based on loading feedback. The main contribution of the protocol is to support fine grain QoS at edges (user QoS) with coarse grain QoS in the backbone network (network QoS).
Pricing-sensitive adaptation can also be provided for in multimedia communications. Thus, the adaptation in the integrated resource negotiation, pricing, and QoS Adaptation Framework for Multimedia Applications is price-sensitive [15]. Here, prices increase during congestion, providing an incentive for applications to adapt their sending rates. The work incorporates a dynamic resource negotiation and pricing protocol, RNAP to enable a user to select from a set of different network services with different QoS characteristics and enable the user and network to dynamically renegotiate the contracted service parameters and price. The authors propose optimization algorithms by which applications (singly, or as part of a multi-application system) can adapt their service requests so as to optimize user satisfaction under the constraint of a fixed budget. Mechanisms are developed within the RNAP architecture for the network to dynamically formulate prices and communicate pricing and charging information to the users.

One of the most interesting developments in multimedia communications has been adaptation based on user requirements. Indeed, integrating user-level expectations with parameters characterising underlying network performance is a problem seldom studied in multimedia streaming, for it attempts to bridge the gap existing between user perceptions of multimedia quality, on the one hand, and the Quality of Service (QoS) with which multimedia is transmitted over the network, on the other. Work in this respect has focused on the effects that different video frame rates have on human satisfaction with the multimedia presentation [16], on the perceptual impact of errors [17], delay [18] and jitter [19], or, alternatively, on the development of metrics for assessing subjective multimedia quality based on models of the human visual system [20].

However, only rarely is such research carried forward in the development of adaptive streaming applications. Accordingly, the QUASAR project [21] exploits human perceptual tolerance to media losses and frame dropping, as does [22]. However, such approaches generally fall short on two counts: firstly, the perceptual tests on which they are based ignore multimedia's infotaiment duality (namely, that all multimedia applications are located somewhere on the information-entertainment continuum); secondly, many of them assume that users of 
distributed multimedia technology have considerable technical skills, which, given the proliferation of the Web, represent the exception rather than the norm today

\section{Quality of Perception in an Adaptive Communication System}

The adaptable protocols and architectures outlined above do not take into account user perception in their functionality. Distributed guaranteed services need to incorporate capabilities for responding to QoP and $\mathrm{QoS}$ changes originating from the user/applications (as a result, perhaps, of the content of the application changing) or the system/network (as a result of changing network conditions), respectively.

In this section we present the case for using adaptable protocols geared towards human requirements in the delivery of distributed multimedia. We have implemented a proof-ofconcept application - a distributed MPEG player - through which multimedia clips can be transmitted over computer networks using a protocol expressly configured for improved QoP delivery. We have thus a user-oriented communication protocol, radically different in concept to legacy protocols which do not take QoP considerations into account. The protocol links user-level QoP to low-level QoS parameters, thereby integrating QoP in an end-to-end multimedia QoS architecture.

\subsection{DRoPS}

The Dynamically Reconfigurable Protocol Stacks (DRoPS) project provides an infrastructure for the implementation and operation of multiple adaptable protocols. The core architecture is embedded within the Linux operating system, is accessible through standard interfaces, such as sockets and the UNIX ioctl (I/O control) system calls, has direct access to network devices and benefits from a protected, multiprogramming environment. The architecture allows additional QoS maintenance techniques, such as flow shaping (to smooth outbursts in traffic), at the user or interface level, and transmission queue scheduling, at the device queue level.
DRoPS-based communication systems are composed of fundamental mechanisms, called microprotocols that perform arbitrary protocol processing operations. The complexity of processing performed by a microprotocol may range from a simple protocol function, such as checksum, to a complex layer of a protocol stack, such as TCP. Like many modular operating systems Linux supports loadable modules. These software objects encapsulate microprotocols, allowing code to be dynamically loaded into a running operating system and executed without the need to recompile a new kernel. A detailed description of DRoPs can be found in [23].

\section{Protocol Stack Construction and Adaptation}

Figure 1 provides an overview of the major system components that form the architecture and defines their interaction. Microprotocols are represented as small circular objects and are divided between two protocols X and Y. A Sub Protocol Controller (SPC) is associated with each connection to represent attributes unique to an individual connection, such as protocol configuration, connection characteristics, user QoS requirements and private protocol data. Figure 1 depicts a protocol stack as an undulating line connecting an SPC to a particular network device. The microprotocols intersected by this line form the stack configuration and are defined by the associated SPC.

Three operations, exclude, include and exchange, are provided to manipulate the configuration of a protocol stack. The exchange operation swaps one or more microprotocols for other(s) to form a modified protocol stack. Thus the effect of this operation is to manipulate the stack configuration, stored within the associated SPC, routing data from subsequent messages through a different set of microprotocols. In addition, each SPC contains an activation field defining the active microprotocols in the current configuration. End points are notified of reconfiguration by explicit control messages sent over either a dedicated channel or piggybacked on protocol data. DRoPS does not supply adaptation policies, it merely provides an infrastructure on which adaptable protocols can be implemented and adaptation policies executed. The heuristic used to implement a policy is arbitrary and may be supplied by an application or a protocol. 


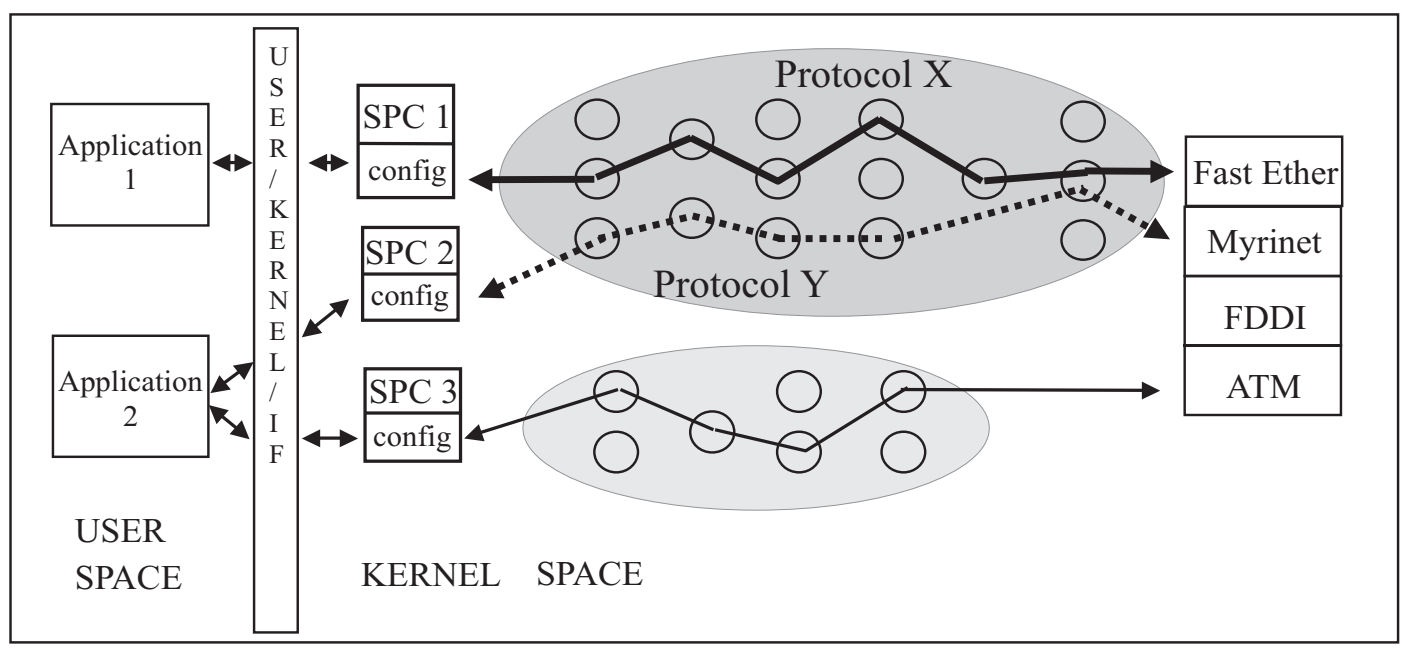

Fig. 1. Relation between DRoPS objects, user applications and operating system.

The conceptual architecture of an adaptation control system can be seen in Figure 2. Adaptation policies form the core of the control system. The shaping function receives required and provided QoS information and ensures these values are bounded and scaled according to the functions that reported them. The shaped statistics are fed to the relevant adaptation policies which output desired protocol configurations. These configurations are then checked against the existing configuration to determine if reconfiguration is necessary and sanity is checked to ensure validity. The result of this processing is a set of include, exclude and exchange commands that cause DRoPS to perform reconfiguration at the relevant end points of communication.

A heuristic control scheme is required to maintain a communication service with a provided QoS closest to that required by an application.
Lookup tables, Boolean logic, fuzzy logic and neural networks are all considered as potential generic control mechanisms. Ultimately, the mechanism used is defined by constraints of code size, processing overhead and generality of solution.

Minimising the difference between required and provided QoS is a non-trivial task. The mapping of provided QoS to protocol configuration is additionally hampered by constraints such as host resources and underlying hardware capabilities. In DRoPS, a neural network adaptation policy controller is used to perform generic protocol adaptation. This choice is motivated by the overhead of one order of magnitude, smaller compared to other heuristics and also because the neural network controller performs generalization. It is thus able to suggest appro-

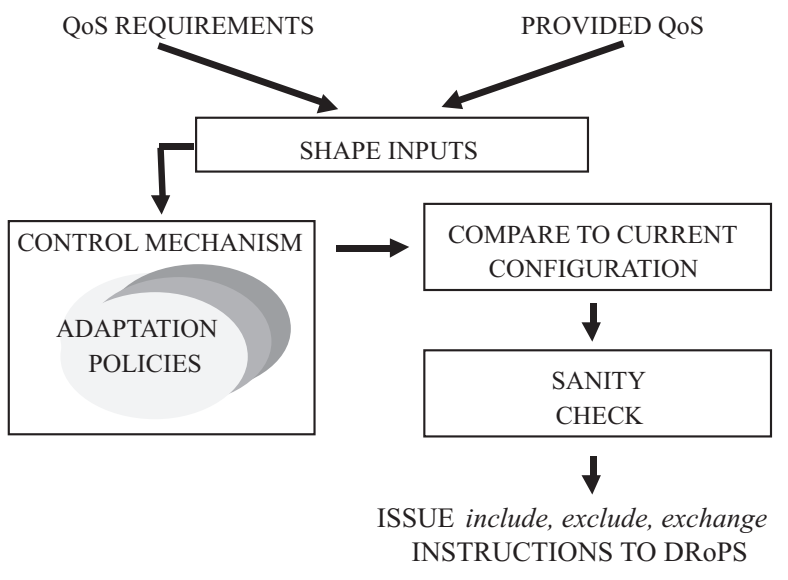

Fig. 2. Generalised structure of the DRoPS adaptation control system. 
priate protocol configurations for unseen scenarios. A feed forward multilayer perceptron (MLP) has been implemented and used to experiment with neural networks as protocol adaptation controllers.

\section{Experimental Study}

We have undertaken an experimental study which has involved testing and comparing the performance, from a QoP perspective, of two legacy protocol stacks, TCP/IP and UDP/IP, with the Reading Adaptable Protocol (RAP). The Reading Adaptable Protocol is a complete communication system developed within the DRoPS framework, composed of multiple microprotocols. Moreover, whilst RAP was specially configured for providing improved QoP in a networked setting, in the first two protocol stacks there is no provision for QoP management.

A total of 12 users, 36 in all, have been tested for each protocol stack. All users were computer literate, had used multimedia applications before and all spoke English well enough to be educated in the medium. The youngest user was 20 years old, while the oldest was 67 . Each user was presented with a set of 12 short (30-45 seconds' duration) multimedia clips in MPEG-1 format. The users were asked a series of 10-12 questions per clip on its informational content and were then asked to rate their perception of the display quality of the clips on a scale of 1-6 (with scores of 1 and 6 representing the worst and, respectively, best perceived qualities possible). The clips were chosen to be as varied as possible, ranging from a relatively static news clip to a highly dynamic rugby football sequence (Table 2). All of them depicted excerpts from real world programmes and thus represent informational sources which an average user might encounter in everyday life.

The clips had a nominal playback rate of $15 \mathrm{fps}$ (a usual frame rate in the case of windowed $352 * 288$ pixels multimedia clips) all with a constant colour depth of 8 bits. Users were not aware of the protocol with which a clip was being transmitted, nor of the associated frame rate and colour depth, with each user being randomly assigned to a particular protocol at the beginning of the experiment. In order to counter

\begin{tabular}{|l|}
\hline VIDEO CATEGORY \\
\hline 1 - Action Movie \\
\hline $2-$ Animated Movie \\
\hline $3-$ Band \\
\hline $4-$ Chorus \\
\hline $5-$ Commercial \\
\hline 6 - Cooking \\
\hline $7-$ Documentary \\
\hline $8-$ News \\
\hline $9-$ Pop Music \\
\hline $10-$ Rugby \\
\hline $11-$ Snooker \\
\hline $12-$ Weather Forecast \\
\hline
\end{tabular}

Table 2. Video Clip Categories.

balance any possible order effects, the presentation order of the clips to the users was varied.

The protocol adaptation controller is implemented via a neural network. User-centric requirements are passed to the control system through DRoPS taking into consideration the QoP-QoS mapping. The associated weight of four QoS parameters (bit error rate, segment loss, delay and jitter) was computed according to the QoP-QoS proportionality and passed on as four inputs to the neural network controller. Based on the devised mapping [9], low, medium and high values for the video, audio and textual components, respectively, of a clip would result in:

$$
Q o P_{I T} \cong B E R+\frac{11}{6} D E L+\frac{10}{6} J I T+\frac{5}{3} S L+\frac{5}{2} S O
$$

Thus, neural network inputs corresponding to the required values for bit error, delay, jitter and loss would have the values $1,11 / 6,10 / 6$ and $5 / 3$ respectively. The remaining four inputs to the controller consisted of appropriately scaled monitored values of the respective QoS parameters, while the four logical groups of outputs of the neural network delineated an appropriately tailored protocol configuration defining the new adapted protocol.

Currently, the neural network-controlled adaptation polices provide a best effort service where the realised QoS is as close as possible to the requested QoS within the current environment. 
Results have shown that the use of such a neural adaptation controller does not contribute significant processing overheads (a mean of $190 \mu \mathrm{s}$, with a variance of $+/-6 \mu$ s for all cases) and is able to reduce the complexity and costs of protocol processing whilst providing a more appropriately configured transport service.

\section{Results}

The two main components of QoP - understanding and assimilation of informational content, on the one hand, and satisfaction, on the other, were both analysed. In order to evaluate the impact of the three different protocol stacks used on a user's QoP associated with the multimedia clips presented in our experiments, a 2way Analysis of Variance (ANOVA) was done. The two independent variables considered were protocol type and clip category.

\subsection{Impact of Transmission Protocol on Quality of Perception (Understanding and Information Analysis and Synthesis)}

Analysis revealed that the particular protocol being used to transmit the multimedia clip significantly impacts upon a user's ability to understand, analyse, and synthesise the informational content of multimedia clips $(\mathrm{F}(2,252)=$ 5.96, $\mathrm{p}<.01)$.

Volunteers who saw multimedia clips transmitted with the UDP/IP protocol stack fared the worst, as far as the percentage of correct answers obtained goes. This is probably because of the unreliable nature of UDP's data transmission mechanism, which results in the display of corrupted data and ignorance of any out of order sequences. The performance of the TCP/IP protocol stack as reliable conveyor of multimedia bit streams closely matches the one obtained using RAP. The reason behind this achievement could very well be the reliable, error-correcting data delivery mechanism associated with TCP/IP. While TCP/IP performs better for relatively static multimedia clips such as Band or Choir, RAP delivers better performance for clips which are dynamic in nature (e.g. Rugby clip), or for those with a multitude of informational sources (e.g. Commercial clip). Moreover, RAP also outperforms TCP/IP for some clips which are mildly dynamic and have an above-average informational load such as a Cooking scene.

Therefore, there are cases - static clips for instance - where the overheads incurred by the neural network policy controller, as well as through reconfiguration in RAP, are not called for. However RAP, does seem to give better performance in the case of mildly or highly dynamic clips, as well as for those which are informationally rich and complex. Thus, in this case, the better QoP provided outweighs the slight overheads associated with the neural network.

\subsection{Impact of Transmission Protocol on Quality of Perception (Satisfaction)}

Our results have showed that user satisfaction varies strongly according to the underlying transmission protocol being utilised $(\mathrm{F}(2,252)=$ $7.463, \mathrm{p}<.001)$.

For ten out of the twelve video categories visualised, the DRoPS-based protocol stack does result in the highest user rating of the associated distributed multimedia presentation quality (Figure 3). This is a consequence of the fact that the adaptable, reconfigurable nature of RAP as well as the QoP-QoS mapping incorporated in it result in RAP having a tailor-made functionality best suited to the application's current needs. Although UDP/IP does have the smoothest playback out of the three protocol stacks employed, the fact that RAP corrects errors (which, moreover, do not therefore propagate in the frame sequence) encountered in the bit stream seems to account for the primacy of this protocol stack over the two others in delivering a consistently high satisfaction rating.

In the case of moderately dynamic clips with above-average and complex (as regards the number of sources) informational load, usage of RAP delivered best overall QoP. The second observation is that user satisfaction does not depend on the particular type of clip being visualised. 


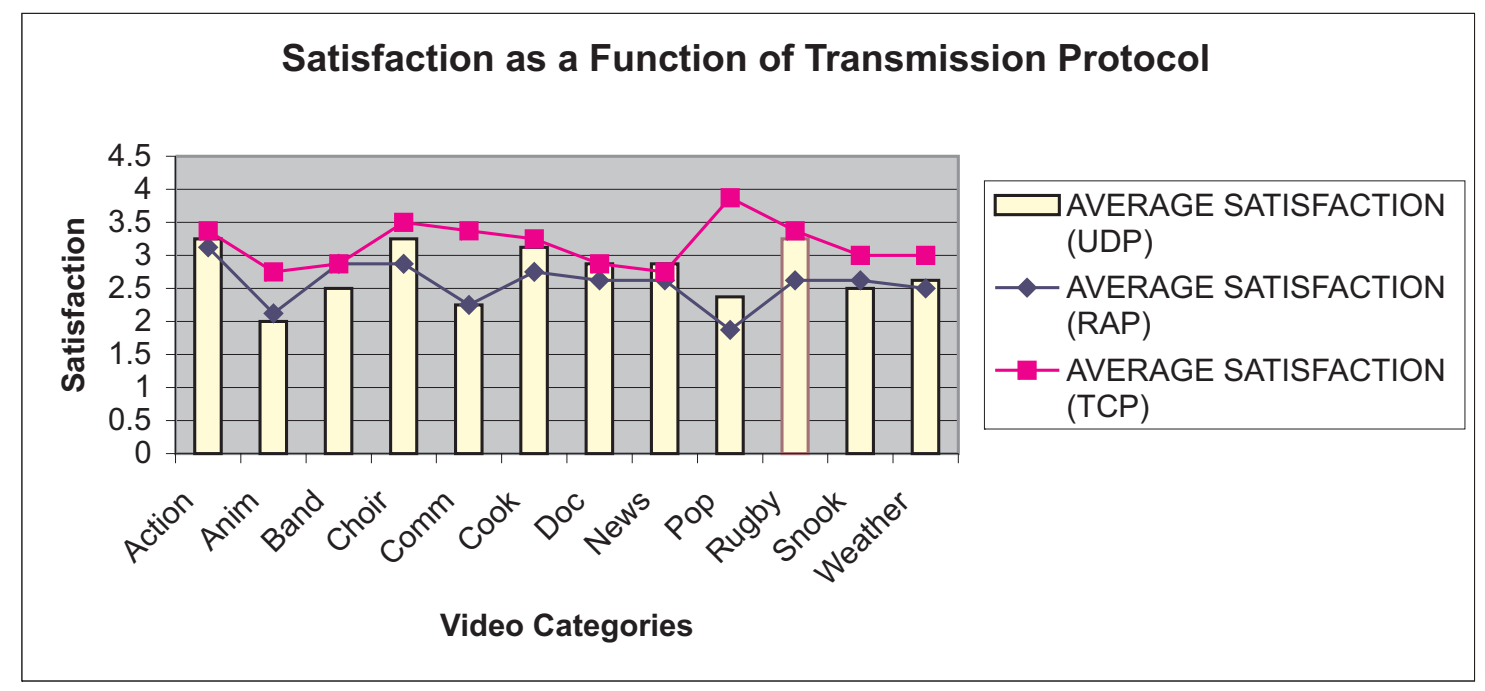

Fig. 3. Variation of QoP (satisfaction) with transmission protocol employed.

\subsection{Standalone vs. Distributed Case}

If one compares the QoP achieved under the three communication systems (TCP/IP, UDP/IP, RAP) to the one obtained in the control (standalone) case, where no protocol overheads were involved, the conclusion to be drawn is that the QoP-QoS mapping does have practical validity. Thus, RAP, which incorporates the mapping, is the only protocol stack out of the three considered, capable of delivering overall QoP results which are not in terms of statistics, significantly different from the ones obtained in the ideal, standalone scenario. Furthermore, as opposed to other attempts [1] [4], to include user perceptual requirements in schemes for end-to-end QoS management, the architecture proposed manages QoP automatically without any need for user input.

In more detail, a 2-way ANOVA is performed pair-wise with the results obtained using each of the three communication protocols, on the one hand, and those obtained in the standalone case, on the other. Differences in the understanding, analysis and synthesis of information contained in the visualised multimedia clips between the standalone case and the case when UDP/IP is used as a protocol stack, are statistically significant $(\mathrm{F}(2,252)=7.596, \mathrm{p}<.001)$. Thus, not only does UDP/IP deliver a lower QoP as far as information assimilation goes, but these differences are statistically significant and are in most probably due to the unreliable nature of the data transmission mechanism of this protocol stack.
In the case of both RAP as well as TCP/IP, the differences in the information assimilation side of QoP delivered under these two protocol stacks and the one obtained in the standalone case are not statistically significant. This observation comes in spite of the fact that the considered QoP values in the standalone case are, generally speaking, higher in absolute terms than those achieved using RAP and TCP/IP. What this shows is the effectiveness of the QoP-QoS mapping incorporated in an adaptable protocol to deliver a QoP whose results do not differ significantly from a statistical point of view to the ideal, standalone scenario.

\section{Conclusions}

The main contributions of our work all stem from our belief that networking research must gravitate towards the requirements of the enduser, whereas the situation commonly encountered in the literature is exactly the opposite. Towards this end, we have outlined a mapping linking user-centric QoP to low-level QoS parameters, thus showing a way of prioritising among QoS parameters so that QoP is optimised. The research has also studied how QoP varies with different protocol stacks. As far as we are aware, not even previous work which investigated user satisfaction with multimedia applications has attempted this, rather focusing on standalone applications. Not only have 
we explored how QoP is affected by two of the most commonly used transmission protocol stacks (TCP/IP and UDP/IP), but we have shown that there is a strong scope for QoP improvements if the QoP-QoS mapping is introduced in a specially tailored adaptable protocol.

We recognize that there are limitations to our work - an in-depth profiling of users could help in understanding QoP better, as could a greater number of participants in the experiments themselves. Nonetheless, our research has opened up exciting avenues for future exploration. An interesting issue would be to investigate how introducing pricing considerations might affect the whole QoP framework. The QoP-QoS mapping needs to be refined. Some applications are used exclusively for informational purposes, others for entertainment, while in between there is a wide range of infotainment applications where there is a combination of the two goals, but even then in varying proportions. Accordingly, how would QoP vary across different scenarios such as teleconferencing, distance education and computer-supported co-operative work? How can QoP be improved by exploiting the Differentiated Service (DiffServ) architecture is another area which has not been investigated yet.

\section{References}

[1] M. Fry, A. Seneviratne, A. Vogel, AND V. WITANA, Delivering QoS Controlled Continuous Media on the World Wide Web, Proceedings of the $4^{\text {th }}$ International Workshop on $Q o S$ (IWQoS), pp. 115-124, Paris, France, 1996.

[2] S. ARdon, R. DeSilva, B. LANDFELdT, AND A. SENEVIRATNE, Total Management of Transmissions for the End-User, a Framework for User Control of Application Behaviour, Proceedings of the $4^{\text {th }}$ International Workshop on High Performance Protocol Architectures (HIPPARCH '98), London, June 1998.

[3] A. Vogel, B. Keherve, G. VON Bochmann, AND J. GECSEI, Distributed Multimedia Applications and Quality of Service - A Survey, IEEE Multimedia, Vol. 2, No. 2, pp. 10-19, Summer 1995.

[4] D. REININGER, D. RAYChaudhuri, AND M. OTT, A Dynamic Quality of Service Framework for Video in Broadband Networks, IEEE Network, pp. 22-34, November/December 1998.
[5] A. Seneviratne, M. Fry, V. Withanana V. SapaPRAMDU, A. RICHARDS AND E. HORLAIT, Quality of Service Management for Distributed Multimedia Applications, IEEE Communication Society, Proceedings of the Phoenix Conference on Computer and Communications, Phoenix, Arizona, USA, 1994.

[6] G. KALKBRENNER, T. PirkMAYER, A. VAN DORNIK, AND P. HoFMANN, Quality of Service (QoS) in Distributed Hypermedia Systems, Proceedings of the Second International Workshop on Principles of Document Processing, April 1994, Darmstadt, Germany.

[7] K. NAhrstedt, A. Hossain, AND S. KANG, A Probe-based Algorithm for QoS Specification and Adaptation, Proceedings of the 4th International Workshop on QoS (IWQoS), pp. 115-124, Paris, France, March 6-8, 1996.

[8] G. GHINEA AND J. P. ThOMAS, QoS Impact on User Perception and Understanding of Multimedia Video Clips, in R. Steinmetz and W. Hall (Eds.), Proceedings of ACM Multimedia '98, pp. 49-54, Bristol, UK, 1998.

[9] G. GHINEA AND J. P. Thomas, Crossing the ManMachine Divide: A Mapping Based on Empirical Results, Journal of VLSI Signal Processing, Vol. 29, No. 1/2, pp. 139-147, 2001.

[10] A. CAmpbell, And G. Coulson., QoS Adaptive Transports: Delivering Scalable Media to the Desktop, IEEE Network, pp. 18-27, March/April 1997.

[11] Yamazaki, T. AND J. Matsuda, Adaptive QoS management for multimedia applications in heterogeneous environments: A case study with video QoS mediation, IEICE trans. Communications, November 1999.

[12] B. BANDrinath, A. FoX, L. KLEINROCK, G. POPEK, P. RETHER, AND M. SATYANARAYANAN, A conceptual framework for network and client adaptation, Mobile Networks and Applications, Vol. 5, pp. 221-231, 2000.

[13] D.C. SCHMIDT, ADAPTIVE: An object-oriented framework for flexible and adaptive communication protocols, Ph.D. Thesis, University of California at Irvine, USA, 1995.

[14] C. LeE, K. W. Tsai And L. YAng, An Adaptive QoS Protocol for Total Network Traffic Management, Int. Symposium on 3rd Generation Infrastructure and Services (3GIS), Athens, Greece, July 2001.

[15] X. Wang And H. SchulzRinne, An Integrated Resource Negotiation, Pricing, and QoS Adaptation Framework for Multimedia Applications, IEEE Journal on Selected Areas in Communications (JSAC), vol. 18, 2000.

[16] G. YadaValli, M. A. MasRY, AND S. Hemami, Frame Rate Preferences in Low Bit Rate Video, Proc. IEEE ICIP, Barcelona, Spain, pp. 441-444, 2003.

[17] S. VARADARAJAN, H. Q. NGO, AND J. SRIVASTAVA, An Adaptive, Perception-Driven Error Spreading Scheme in Continuous Media Streaming, Proc. ICDCS 2000, Taipei, Taiwan, pp. 475-483, 2000. 
[18] A. Bouch, A. KUCHINSKY And N. BHATti, Quality is in the eye of the beholder, Proc. CHI 2000, The Hague, The Netherlands, pp. 297-304, 2000.

[19] K. Hikichi, H. Morino, S. Matsumoto, Y. YASUDA, I. ARIMOTO, M. IJUME AND K. SEZAKI, Architecture of Haptics Communication System for Adaptation to Network Environments, Proc. IEEE International Conference on Multimedia and Expo, Tokyo, Japan, pp. 744-747, 2001.

[20] Y. Sermadvi, M. A. Masry and S. S. Hemami, MINMAX Rate Control with a Perceived Distortion Metric, Proc. SPIE - Visual Communications and Image Processing, San Jose, California, USA, January 2004.

[21] C. Krasic, J. Walpole And W. Feng, QualityAdaptive Media Streaming by Priority Drop, Proceedings NOSSDAV'03, Monterey, California, USA, pp. 307-310, 2003.

[22] A. MAHANTI, D. L. EAGER, M. K. VERnON AND D.J. SUNDARAM-STUKEL, Scalable on-demand media streaming with packet loss recovery, IEEE/ACM Transactions on Networking, Vol. 11, No. 2, pp. 195-209, 2003.

[23] G. Ghinea, J. P. Thomas, And R. S. Fish, Mapping Quality of Perception to Quality of Service: the Case for a Dynamically Reconfigurable Communication System, Journal of Intelligent Systems, Vol. 10, Nos. 5/6, pp. 607-632, 2000.

$$
\begin{array}{r}
\text { Received: July, } 2004 \\
\text { Revised: August, } 2004 \\
\text { Accepted: August, } 2004 \\
\\
\text { Contact address: } \\
\text { Gheorghita Ghinea } \\
\text { Brunel University } \\
\text { Uxbridge } \\
\text { Middlesex, UB8 3PH } \\
\text { UK } \\
\text { e-mail: george.ghinea@brunel. ac.uk } \\
\text { Johnson P. Thomas } \\
\text { Department of Computer Science } \\
\text { Oklahoma State University } \\
\text { Tulsa, OK 74106 } \\
\text { USA } \\
\text { e-mail: jpt@okstate. edu }
\end{array}
$$

GHEORGHITA GHINEA is a lecturer in the Department of Information Systems and Computing in Brunel University. His research interests include multimedia networking, perceptual quality issues, as well as ubiquitous computing.

JOHNSON P. THOMAS is an associate professor in the Department of Computer Science at Oklahoma State University. His research fields span the application of artificial intelligence to multimedia, security and networking. 